\title{
Exploring the Transition from Preventive Maintenance to Predictive Maintenance Within ERP Systems by Utilising Digital Twins
}

\author{
Liam DAMANT ${ }^{\mathrm{a}}$, Amy FORSYTH $^{\mathrm{a}, 1}$, Ramona FARCAS ${ }^{\mathrm{a}}$, Melvin VOIGTLÄNDER ${ }^{\mathrm{a}}$, \\ Sumit SINGH ${ }^{\mathrm{a}}$, Ip-Shing Fan ${ }^{\mathrm{a}}$, Essam SHEHAB ${ }^{\mathrm{b}}$ \\ ${ }^{a}$ Manufacturing Department, School of Aerospace, Transport and Manufacturing, \\ Cranfield University, Cranfield, Bedford UK \\ ${ }^{b}$ Mechanical and Aerospace Engineering Department, School of Engineering and \\ Digital Sciences, Nazarbayev University, Nur-Sultan, Kazakhstan
}

\begin{abstract}
Over the years, there has been an advancement in how manufacturing companies conduct maintenance. They have begun transitioning from Preventive Maintenance (PM) to Predictive Maintenance (PdM). With the introduction of technologies such as Digital Twin (DT), Internet of Things (IoT), and Intelligent Manufacturing (IM), the world is rapidly changing, thus allowing companies to optimise existing processes, products and reduce costs. The existing literature offers limited investigations and best practices in the end-to-end optimisation for maintenance transformation. The current paper intends to explore (a) the transition from PM to PdM and (b) the utilisation of DTs and IM for maintenance optimisation. The paper articulates the scope and features of end-to-end maintenance optimisation for asset uptime and cost benefits. The findings can help industries understand the introductions and advancements of technologies for predictive maintenance and end-to-end optimisation with the benefit of investigating and illustrating how companies can move forward.
\end{abstract}

Keywords. Predictive Maintenance, Preventive Maintenance, Digital Twin, Internet of Things, Enterprise Resource Planning (ERP), Artificial Intelligence, Intelligent Manufacturing, Optimisation, Transdisciplinary

\section{Introduction}

The advancements brought about by Industry 4.0 have enabled the integration of digital technologies in many businesses. Exploring this digital integration uncovers new improvement opportunities and challenges. However, the latest technologies are not sufficiently understood by many companies yet. Manufacturers do not create the right conditions for their optimal implementation and thus do not fully exploit the inherent potential. When transitioning from PM to PdM within the manufacturing industry, there are multiple advanced technologies that companies need to consider for their production

\footnotetext{
${ }^{1}$ Corresponding Author, Mail: Amy.L.Forsyth@cranfield.ac.uk.
} 
assets. It is almost certain that companies embracing the digital transformation will generate insights from their processes they did not have before.

This paper investigates how advanced technologies enable the transition from PM to PdM and its integration into a companies' ERP system. It emphasises that in a valuedriven maintenance approach, the benefits of PdM must outweigh transition costs coming with it to generate a positive business case. A potential business case is reinforced by an innovative end-to-end optimisation that allows the inclusion of all relevant data for holistic optimisation and decision-making enabled by the use of PdM. This paper, therefore, adds to the literature while it provides valuable and actionable guidance for manufacturers considering the implementation of PdM by combining and relating different transdisciplinary technologies and methods. Practical implications when applying DT and IM technology to a PdM approach are elaborated. Potential stumbling blocks and possible responses to them are also discussed to provide a complete picture. A developed PdM implementation roadmap and defined system requirements round up the approach and facilitate the implementation process.

\section{Total Cost of Maintenance}

A successful business is primarily defined by a positive Return on Investment (ROI). In the present case, manufacturers use the ROI to measure the value generated through maintenance activities (see Table 1) and assess the success of single measures. The ROI depends on the scale of operations, which assets are critical to maintaining and how much a company spends. To achieve a positive return, measurable and nonmeasurable factors must be identified. Subsequently, companies can identify the necessary technologies for the transition from PM to PdM. Once a company applies the costs of new technologies to its circumstances, it can effectively compare the potential cost saving and the impact of maturing the systems and equipment with the investment costs to assess whether the transition to $\mathrm{PdM}$ is economically viable for them. Figure 1 illustrates the potential programme savings for PdM.

Table 1. Predictive Maintenance Programme Savings.

\begin{tabular}{|c|c|c|c|c|c|c|}
\hline Increase asset availability & $5-15 \%$ & & $9 \%$ & V & $30 \%$ & \\
\hline Reduce Maintenance Cost & $18-25 \%$ & $25-30 \%$ & $12 \%$ & $10-40 \%$ & $50 \%$ & $25 \%$ \\
\hline Reduction in breakdowns & & $70-75 \%$ & & & $55 \%$ & $70 \%$ \\
\hline Reduction in downtime & & $35-45 \%$ & & $50 \%$ & & $50 \%$ \\
\hline Increase in production & & $20-25 \%$ & & V & & \\
\hline Reduce H\&S and quality risks & & & $14 \%$ & $10-20 \%$ & & \\
\hline Extend lifetime of aging assets & & & $20 \%$ & $3-5 \%$ & & \\
\hline Increase employee morale & & & & V & & \\
\hline Repair and overhaul time & & & & & $60 \%$ & $12 \%$ \\
\hline Spare parts inventory & & & & & $30 \%$ & \\
\hline Return of investment & & 10 & & & & \\
\hline Cut unplanned outage & & & & & & $50 \%$ \\
\hline Reduction capital investment & & & & & & $3-5 \%$ \\
\hline References & $\begin{array}{c}\text { (Morgan Cox et al., } \\
\text { 2017) }\end{array}$ & $\begin{array}{l}\text { (Gutenberg- } \\
\text { technology, 2019), } \\
\text { (U.S Department of } \\
\text { Energy's Federal } \\
\text { Energy Management } \\
\text { Program,2004) }\end{array}$ & $\begin{array}{c}\text { (Kupervas Amir, 2019) } \\
\text { (Al Multiple, 2020) }\end{array}$ & $\begin{array}{c}\text { (AI } \\
\text { Multiple, } \\
2020)\end{array}$ & $\begin{array}{l}\text { (SPD } \\
\text { group, } \\
2020)\end{array}$ & $\begin{array}{l}\text { (IBM, } \\
2017)\end{array}$ \\
\hline
\end{tabular}

Manufacturers need to consider the following aspects to estimate the cost of maintenance and its associated losses: 
- Direct maintenance and repair costs: labour and materials

- Indirect costs: downtime, lost sales due to quality/delays, low quality (rework/scrap), and energy usage.

Additionally, the cascading consequences which are linked to subsequent losses should also be accounted for, as outlined by [1]:

- Overtime due to improper balance of work, wrong people for the job

- Spares maintenance due to poor management, low ordering or missing spares

- Downtime of downstream machines

- Downtime on the capital and labour cost due to maintenance

- Low quality on sales and delivery, but also the losses due to rework/scrap.

Whilst the significant effect of $\mathrm{PdM}$ is being estimated using a range of metrics when it comes to quantifying the maintenance, this is not well documented across organisations. The NIST publication examines asset maintenance cost and provides comprehensive guidance regarding the data needed for making discrete estimates. Understanding and quantifying the different data enables optimal maintenance decision-making, including choosing between PM and PdM, selecting resources and a spare part management methodology. One method for measuring maintenance cost is using the input-output data, alongside particular equations for calculating the overall maintenance cost [1]. A prerequisite to estimating maintenance costs is gathering the correct data, which means data is accurate, complete, and appropriately provided [2]. The information in the ISO standard [2] is recommended for data collection during the whole life cycle of an asset until the asset is decommissioned. Digital manufacturing using an IoT platform as an interface can facilitate the data gathering of various differing sources.

\section{Transition from Preventive Maintenance to Predictive Maintenance}

Organisations are focusing on ensuring that their physical assets are productively running and functioning correctly to reduce costs. Maintenance activities generally include functional testing, examination, servicing, repairs, or overhaul of assets to restore or maintain the assets operational condition throughout its working lifecycle [12]. PM is carried out regularly while the asset is still in a working condition to prevent sudden breakdowns. PdM uses various data sources to determine when a machine or component within an asset will fail, and it also incorporates asset data from the environment in which it operates and the associated processes and resources that interact with the asset. PdM does not tell when an asset will fail but forecasts the future performance and analyses these cycles to define the probability of failure based on various parameters. This methodology, therefore, requires Predictive Modelling, of which there are many different approaches. Fuzzy Logic, Regressions and Decision Trees are commonly used forms of logic when building the predictive model. The selection depends on the machine's application [3].

PdM relies on a live data feed coupled with analytics to determine a flexible schedule because it changes based on the input data stemming from the asset. Therefore, predictive analytics must be performed on the data received from that particular asset to define this new schedule. Companies must understand at what point in the process flow and what method of analytics to use. Figure 1 illustrates the input to output flow. 


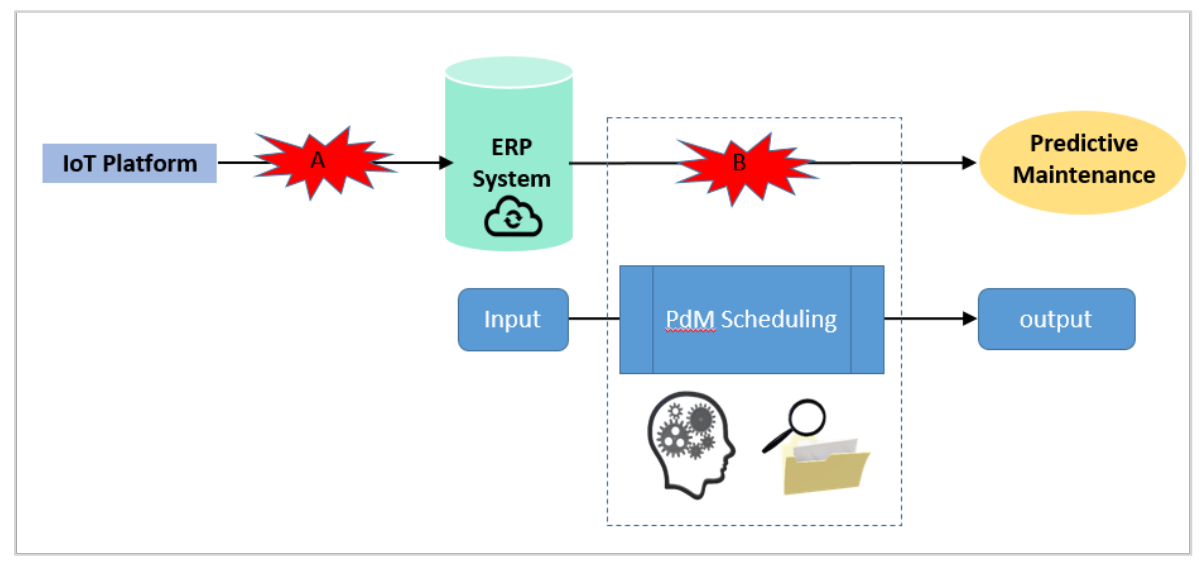

Figure 1. Predictive Maintenance Solutions.

The data input is shared via industrial IoT from the asset to the companies' ERP system. It is this integration with ERP that enables the business decisions, including PdM, to be executed. As shown, there are two logical points in the process flow highlighted as "A" and "B" where a manufacturer can perform predictive analytics. Point "A", which is closer to the source of data collection, offers the potential to utilise ML and Industrial Edge computing strategies [4], while point "B" requires the ERP system to be capable of predictive analytics. Although both methods prove capable of predictive analytics, the method selected must be suitable for any specific business context.

It is suggested that there are multiple methods to achieve this analytic process. ML, Industrial Edge Computing, Algorithmic Programming and AI prove to be some of the most appropriate ways of achieving this. These advanced technologies require a good understanding of reliability engineering and data science to provide useful maintenance information. To act upon this information within a maintenance programme, integration with ERP becomes necessary. Integrating analytics data into ERP demands additional functionality of the system, and it becomes apparent that the integration is highly individual and dependent on the products, assets and system landscape of every manufacturer. This can make the implementation more complex and costly. ERP systems tend to be generic in their design, so often, parts of the optimisation are not economically realised in an ERP system. Therefore, manufacturers must find ways to design and implement an upstream application that feeds into an existing ERP system. Ultimately, it is down to the ERP suppliers to create the functionality to integrate and process industrial IoT data.

\subsection{PdM Roadmap}

Performing predictive analytics is one section of the puzzle when transitioning towards PdM. Any organisation seeking to establish a PdM regime should follow a defined strategy outlining the requirements that define which activities to undertake. Before implementing any strategy and incurring the associated cost expenditure, the organisation should first assess its current maintenance capabilities and justify its business requirement to transition to PdM. Table 2 outlines an appropriate roadmap. The outlined scope of the implementation roadmap would typically require the organisation to hold a prerequisite of diagnostic condition-based monitoring capabilities. The end of 
the roadmap forms the foundation of prescriptive maintenance, should that be a future aspiration of the organisation.

Table 2. Predictive Maintenance Implementation Roadmap.

\begin{tabular}{|c|c|c|c|}
\hline Strategy & Activity & Requirements & Enablers \\
\hline Step 1 & Identify asset(s) for PdM & $\begin{array}{c}\text { Define the scope of Programme } \\
\text { Complexity/value evaluation of } \\
\text { asset }\end{array}$ & $\begin{array}{l}\text { Total cost of } \\
\text { Maintenance } \\
\text { Evaluation }\end{array}$ \\
\hline Step 2 & Establish data sources & $\begin{array}{c}\text { Acquire Software as a Service } \\
\text { tools, software, and } \\
\text { computational infrastructure }\end{array}$ & $\begin{array}{l}\text { Edge Computing } \\
\text { (Optional) }\end{array}$ \\
\hline Step 3 & $\begin{array}{c}\text { Analyse failure modes (e.g. } \\
\text { Failure Mode Effects and } \\
\text { Analysis) }\end{array}$ & $\begin{array}{c}\text { Define prognostic logic of } \\
\text { asset(s), Define prognostic logic } \\
\text { of asset(s) }\end{array}$ & $\begin{array}{l}\text { Asset Lifecycle } \\
\text { Management, Digital } \\
\text { Twin }\end{array}$ \\
\hline Step 4 & $\begin{array}{l}\text { Select and implement } \\
\text { condition-based monitoring } \\
\text { techniques and equipment }\end{array}$ & Sensors and data sharing & IoT; Cloud \\
\hline Step 5 & $\begin{array}{l}\text { Produce predictive } \\
\text { algorithms and Key } \\
\text { Performance Indicators (e.g. } \\
\text { Remaining Time-to-Failure) }\end{array}$ & $\begin{array}{l}\text { Creation of ML predictive model } \\
\text { (e.g. Unsupervised Learning, } \\
\text { Supervised Learning, } \\
\text { Reinforcement Learning) } \\
\text { Typically requires data science } \\
\text { consultants. }\end{array}$ & $\begin{array}{l}\text { Machine Learning, } \\
\text { Artificial Intelligence }\end{array}$ \\
\hline Step 6 & $\begin{array}{l}\text { Generation and deployment } \\
\text { of a predictive maintenance } \\
\text { schedule }\end{array}$ & $\begin{array}{c}\text { Work Order Tracking, Material } \\
\text { Resource Planning, Labour } \\
\text { allocation }\end{array}$ & $\begin{array}{c}\text { ERP, Manufacturing } \\
\text { Execution System } \\
\text { (MES) }\end{array}$ \\
\hline
\end{tabular}

\subsection{System Functionality Requirements for Maintenance Optimisation}

Maintenance optimisation has different system requirements for transitioning from PM to PdM, especially when using a DT. First, companies must fulfil particular prerequisites to implement these technologies. Secondly, the enterprise systems must be able to use the generated outputs of the PdM algorithms and DT applications. If, for example, the PdM algorithm generates an optimised maintenance schedule (as shown in Figure 3 ) and the maintenance scheduling application (within the ERP or MES) is technically unable to schedule the maintenance accordingly, the effect equals zero. Therefore, a company considering the transition to PdM must understand if its existing systems are capable of the shift or if they must be updated, extended, complemented, or replaced. Bumblauskas et al. [5] have identified multiple gaps in information systems literature for asset management. They outline that research on the usage of information systems for maintenance cost control and optimisation is hard to find. Moreover, they highlight that available research papers do not define requirements or specifications for asset management and maintenance systems in publicly disclosed form. This emphasises the necessity of the present paper and further research. There are three major functional requirements in this context:

1. The system must be able to collate and store the necessary data

2. The system must be able to process the data and make use of it

3. The system must be able to generate actionable outputs from the generated insights 
Decision Support Systems are a viable solution for optimisation problems within complex maintenance systems [6]. However, decision-making approaches are seldom part of an enterprise information system [7]. This makes the implementation of an optimisation approach much more complicated. Manufacturers must deploy an optimisation (sub-)system and achieve integration into the existing maintenance scheduling module. If a company is keen to automate its maintenance decisions, it must provide the same data and functionalities to the algorithm as a maintenance employee typically has available.

\section{Digital Twin and Intelligent Manufacturing for Maintenance Optimisation}

PdM relies on the use of products/equipment to optimise maintenance operations. The transition of maintenance is accelerating across industries through the emergence of IoT, whilst utilising DT as a critical enabler for PdM for high-value or high-risk assets, such as aircraft turbines, as airlines' asset. In a short definition, DT is a visual representation of a physical asset or object across its lifecycle, and this is supported by different enablers, including ML algorithms, IoT and AI [8]. DT performs real-time analysis and optimisation. Companies can perform this through the income of real-time data, rather than historical data, which upgrades and optimises the virtual model of the actual asset. This improves efficiency and facilitates the reduction of inconsistencies through the ability to forecast behaviour and verify changes virtually before impacting the real asset. DT can optimise assets, products and processes through its ability to link vast amounts of data to fast simulation. Realistic models allow improvements to performance, increase the quality, and allow product failure to decrease.

A spin on the technology is the presence of multiple classifications of a DT. It has been identified that there are three dominant dimensions to classify the DT. Figure 2 illustrates the dimensions as the hierarchical level, lifecycle phase, which allow 252 combinations to be formed [9]. It also presents a new understanding that DT can be different in many ways, depending on which combination is used with limited overlap.

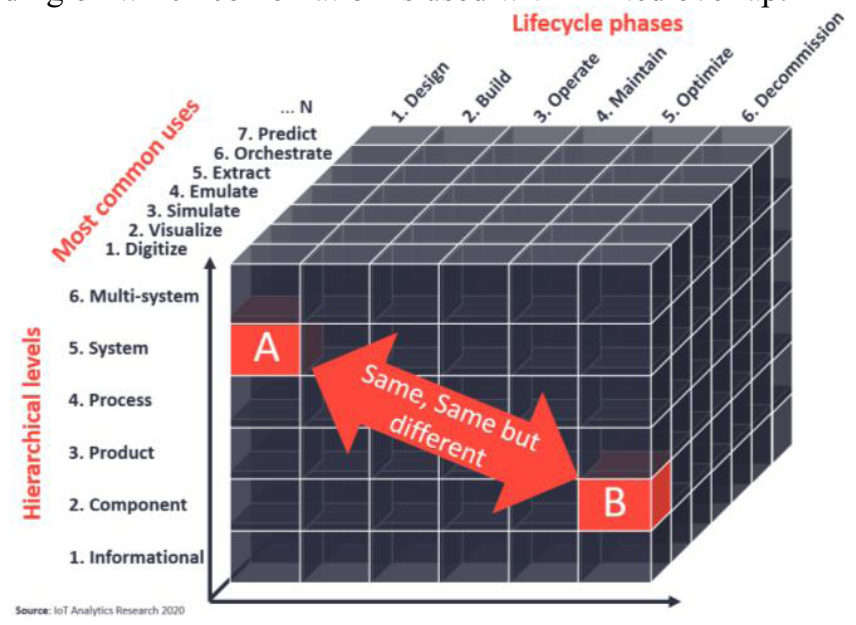

Figure 2. Digital Twin Classifications Cube [9]. 
Introducing IM through IoT and Edge Computing is at the forefront of production optimisation, thus enhancing PdM. The concept of IM has the purpose of optimising production and product transitions using advanced information and technologies in manufacturing. Although this is the next level transformation, companies need to consider the challenging transition due to lack of funding, maturity, and knowledge of advanced technology. IM can be introduced and implemented through utilising multiobjective optimisation algorithms alongside a customised design and other technologies. This enhances the design for maximum performance and the application of PdM. IM enables better productivity and quality with increased flexibility and minimal costs. When looking at the enablers of IM, it is essential to understand how they enhance the maintenance, manufacturing, and synergies to form a powerful front. DT optimises the physical objects based on models that have been mapped and hold a description of elements, whilst IoT facilitates the optimisation of production resources by converting them into smart manufacturing objects [10]. Additionally, Edge Computing increases analytics speed and maximises maintenance penetration, therefore enhancing the production line. Ultimately, for movements towards IM, it is vital to work towards a closed-loop to complete tasks that require intelligent equipment; the movement allows specific tasks to be focused on and applied to actual production, which provides advanced optimisation of individual components. IM is a step in the right direction and will reduce the cost of maintenance for applying manufacturers.

Whilst being promised optimisation with slicker maintenance, companies need to understand that it is virtually impossible or economically viable to incorporate every operating and breakdown mistake within a DT. However, if a company witnesses an unknown situation within a production asset, it can quickly receive support from the supplier. Here, the DT facilitates data transmission from the company to the supplier. Additional information from the DT regarding the location of an asset and parts within the asset provides more detailed work instructions making the maintenance work more efficient and less expensive.

\section{End-to-End Optimisation}

The end-to-end optimisation of processes does not only become available through digital advancements; it also becomes one of the crucial points for successful implementation. If companies spend extensive amounts on a PdM approach and do not integrate it into the overall process, it can hardly cover the investment costs. However, if a company can funnel all process components and the generated data into a single source of truth, it can optimise the whole system and lead to an even more significant financial improvement. By linking all relevant system parameters in a single model focusing on optimising a few selected outputs, asset managers can identify the parameters with the highest impact on the inputs. They can determine which part of an asset causes the highest downtime of a system. Most of the maintenance literature in manufacturing-related fields is concerned with developing analytical models of the actual maintenance [11]. There is a lack of literature and research investigating the here discussed end-to-end optimisation. While the extension of every asset's lifetime is indeed crucial, companies should not neglect the overall optimisation. A door opener for this is that with the introduction of the PdM, the exact timing of the maintenance of individual assets becomes variable, which Figure 3 vividly illustrates. As opposed to PM, companies applying PdM have a specific time 
window to schedule the maintenance work for every single asset. If available, this information can be enriched with numerous further data, for example, from a maintenance bidding and quotation system. Suppose a company can include this within a multiple-input-multiple-output (MIMO) optimisation approach related to the outputs mentioned, the maintenance for each asset can be scheduled to maximise the asset uptime while reducing costs. MIMO approaches are highly complex; however, it offers companies an entirely new scope for asset management.

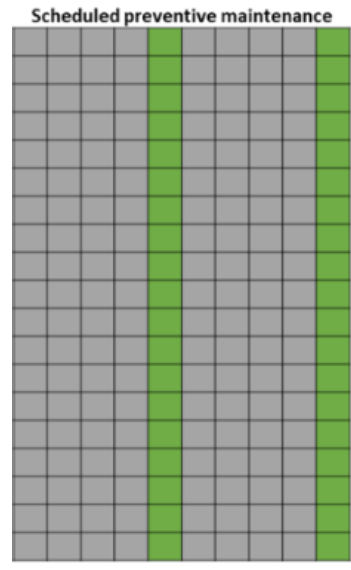

Production

Maintenance

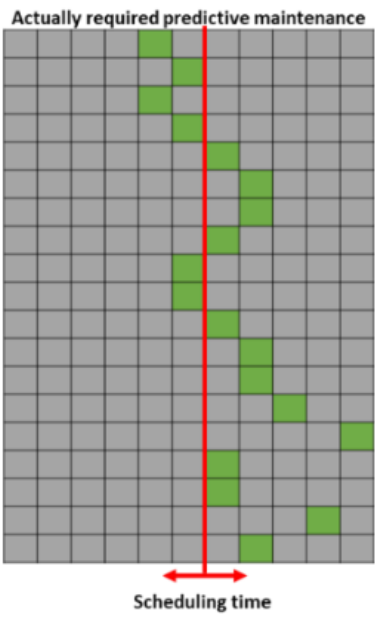

Figure 3. Change of the Maintenance time between PM and PdM.

A further optimisation potential is coming from the assessment of interdependencies of different production departments. If two departments have a separate maintenance budget, but their work is interdepending, the management should assess how a breakdown in the upstream department impacts the downstream department. By having this information interlinked, the management can make a holistic decision that reduces the costs of the whole organisation, not of a single department.

End-to-end relates to the value chain of a company and the lifecycle of a production asset. The Bill of Materials (BOM) is used across a lifecycle consisting of product development, manufacturing, and servicing. Figure 4 illustrates the ideal process of maturing the BOM within the lifecycle and derives from one step to the next. Within this report, the as-maintained BOM is the target of a successful movement on PdM and DT. Whilst transitioning to PdM, it is critical to ensure the as-maintained BOM of the asset is kept accurate and up to date. It must include information about the parts of a machine when they have been maintained. If the BOM is not accurate or up to date, then the DT is effectively no longer one as the actual and virtual models differ. This circumstance can increase the risk of failures and lead to a misprediction of maintenance demand. A challenge here occurs when an unexpected breakdown happens, which is indeed possible under PdM. In this case, every part must be checked for damages and be aligned with the DT to ensure accurate predictions. 


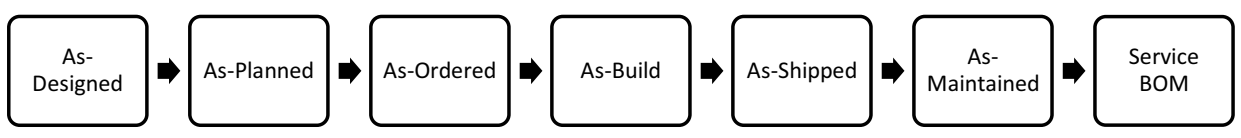

Figure 4. Bill of Materials Lifecycle.

\section{Discussion and Conclusions}

The digital transformation can feel overwhelming for an organisation, especially for those with low digital maturity. PdM and DT approaches are highly complex. Both represent systems that have not been modelled to this level of detail before. Consequently, there is a risk that employees and managers cannot comprehend the generated outputs. This can jeopardise the whole endeavour if employees do not act in accordance with the model. Therefore, it is crucial to commence the described end-to-end preparation and optimisation before companies implement PdM and DT to ensure that every part of the approach is understood.

An ERP system builds the backbone of every large manufacturing organisation. The high complexity and design of an ERP system are widely known to be a significant challenge in the industry. Thus, manufacturers should review every approach in changing or adapting the ERP in an overall context. A successful ERP integration is essential to realise the benefits of the proposed end-to-end optimisation.

Although PdM offers massive potential within asset management, further business operations are considered within this equation. To achieve predictive business operations, including maintenance, integrating live data into management systems is critical. Predictive Analytics within ERP systems requires complex configuration. It seems the future of successful predictive maintenance applications requires ERP systems to receive this data in an analysed state. New exciting technologies capable of ML, such as Edge Analytics and Edge Computing, enabled through IoT and DT technology, appear to be the answer, performing predictive analytics closer to the data source before ERP integration. The report illustrates IM in this context and how it can use DT and other identified enablers to transform manufacturing across industries. While it does not answer all questions at this stage, it provides practitioners with the necessary insights to commence the transition towards PdM.

This paper provides visibility into how integrating different systems and data allows a transition into PdM and ensures that essential steps are considered before commencing the transition. PdM brings massive benefits to an organisation by reducing the breakdowns of machines. When integrated adequately within the end-to-end process, manufacturers can achieve additional advancements. PdM adds more complexity to the maintenance approach of a manufacturer, but if implemented correctly, it also provides an enhanced ability to simulate the companies performance in the real world and act accordingly. The gained flexibility, agility, and increased asset uptime can constitute a competitive edge in an everchanging market environment. 


\subsection{Outlook and Future Research}

The initial thought for the future is understanding whether factories will function entirely autonomously, meaning there would not be any employees within the production line. This environment would minimise human interaction unless it were for maintenance, repairs, base checks, or servicing. This outlook allows the researcher to focus on the linkage between humans and machines to understand how they augment and feed into each other. When machines and robots are not limited to a fixed location anymore, the DT technology will become even more essential. DT, IoT and AI work together to enable advanced and Intelligent Manufacturing. With this advancement being forecasted, understanding how DT data and external data interrelate can add additional strength. The digitalisation of prognostic asset data that is integrated successfully through the concepts detailed within this paper does not just provide the benefit of business decision making but paves the way for automation. The foundations set out by the required systems and technologies needed to achieve PdM can potentially allow autonomous feedback loops. ML can increase its accuracy with increasing time drawing on Deep Learning and AI concepts as the predictive model learn what a 'good' output looks like compared to its collected data set. Should these practices advance further in development and the benefits exceed the costs, there is a high chance manufacturers establish Deep Learning applications that are capable of autonomous business intelligence decisions.

\section{References}

[1] N.N., The costs and benefits of advanced maintenance in manufacturing. NIST, Gaithersburg, MD, 2018.

[2] N.N., Petroleum, petrochemical and natural gas industries - Collection and exchange of reliability and maintenance data for equipment, ISO, 2016.

[3] N.N., Predictive Modeling. MathWorks Inc, 2020, Accessed: 16.11.2020. [Online] Available: https://www.mathworks.com/discovery/predictive-modeling.html

[4] N.N., What is Industrial Edge Computing and why is it improtant?, Exorint, 2020, Accessed: 20.11.2020 [Online] Available: https://bit.ly/2IhgEvk

[5] D. Bumblauskas, D. Gemmill, A. Igou and J. Anzengruber, Smart Maintenance Decision Support Systems (SMDSS) based on corporate big data analytics, Expert Systems with Applications, 2017, pp. 303-317.

[6] J. Bokrantz, A. Skoogh, C. Berlin and J. Stahre, Maintenance in digitalised manufacturing: Delphi-based scenarios for 2030, International Journal of Production Economics, 2017, pp. 154-169.

[7] M.-V Shcherbakov, A.-V. Glotov and S.-V. Cheremisinov, Proactive and Predictive Maintenance of Cyber-Physical Systems, Studies in Systems, Decision and Control, 2020, Vol. 259, pp. 263-278.

[8] A. Stanford-Clark, E. Fran-Schultz and M. Harris, What Are Digital Twins, 2019, Accessed: 26.11.2020 [Online] Available at: https://developer.ibm.com/technologies/iot/articles/what-are-digital-twins

[9] P. Scully, How the world's 250 Digital Twins compare? Same, same but different, Iot-analytics, 2020. Accessed: 10.12.2020 [Online] Available: Iot-analytics.com

[10] R.-Y Zhong, X. Xu, E. Klotz, and S.-T Newman, Intelligent Manufacturing in the Context of Industry 4.0: A Review, Engineering, 2017, Vol. 3(5), pp. 616-630.

[11] B. He, and K.-J Bai, Digital twin-based sustainable intelligent manufacturing: a review, Advances in Manufacturing, 2021, Vol. 9, pp. 1-21.

[12] A.Smith and G. Hinchcliffe, RCM Gateway to World Class Maintenance, Elsevier ButterworthHeinemann, San Fransico, 2004. 